Radiographic, Haemodynamic and Biochemical Finddings Related to the National Heart and Lung Institute's Urokinase Pulmonary Embolism Trial

A. J. JOHNSON, F. STREULI, AND T. HYERS (Department of Medicine, New York University Medical Center, New York) (National Blood Resource Branch Program, National Institutes of Health, Md., from the Urokinase Pulmonary Embolism Trial Study Group)

Eighty-two randomized patients with pulmonary embolism of five days' or less duration were treated with urokinase (UK), 2000 CTA units/lb body weight followed by $2000 \mathrm{CTA}$ units/lb/hr for 12 hours. Results were compared with 78 control patients treated with 75 units of heparin/lb followed by continuous infusion of 10 units $/ \mathrm{lb} / \mathrm{hr}$ for the same period. All patients were then placed on heparin and, subsequently, on anticoagulants, 24 hours after the initiation of therapy. Resolution was significantly greater in the UK group as shown by repeat pulmonary angiography (critical ratio ${ }^{1}$ of 7.8 and $\mathrm{RBO}^{2}$ of $2.9 \times 10^{-13}$ ). Lung scan changes were also significant but of less magnitude (critical ratio of 3.3 and $\mathrm{RBO}$ of $5 \times 10^{-2}$ ). The differences between the urokinase- and heparin-treated groups diminished progressively until day seven of therapy when scan resolutions were the same. Significant differences were also observed in right-sided haemodynamics and in total pulmonary resistance, but not for changes in cardiac output or A-V $\mathbf{0}_{2}$ extraction. Pulmonary angiography and right-sided pressures showed greater differences in patients with massive emboli than in those with submassive emboli. The treatment effect, by lung scan, was significant for pulmonary emboli less than 48 hours old but not for older emboli.

Biochemical data on plasma samples from urokinase-treated patients also showed highly significant changes: the mean pre- and postinfusion plasma levels for plasminogen decreased from $2 \cdot 12$ to 0.60 CTA units $/ \mathrm{ml}$, and for fibrinogen from 515 to $268 \mathrm{mg} / 100 \mathrm{ml}$ while the mean of the whole blood euglobulin lysis times changed from $200 \mathrm{~min}$ before infusion to $16 \mathrm{~min}$ after infusion. Samples from heparin in treated controls showed little or no change. Assays for circulating urokinase revealed about 50 CTA units/ml during the infusion period.

In view of these highly significant clinical and biochemical data, the correlation coefficients were calculated for angiographic changes (toward normal) after infusion of urokinase vs. the biochemical (fibrinolytic) changes in the patient's plasma or the

\footnotetext{
${ }^{1}$ Critical ratio $=\frac{\text { treatment difference }}{\text { standard error }} 2 \cdot 5=$ borderline, $3 \cdot 0=$ fully significant.

${ }^{2} \mathrm{RBO}=$ relative betting odds
}

haemodynamic changes over the same time period. Correlation coefficients for the various parameters were: scan 0.32 , pulmonary artery pressure 0.19 , right atrial pressure $0 \cdot 27$, fibrinogen $0 \cdot 03$, plasminogen 0.02 , and change in whole blood euglobulin lysis time 0.00 . The extraordinary lack of correlation between embolus resolution and degree of activation of the fibrinolytic system led us to conclude that at this dosage of urokinase the patients' clinical response probably depends primarily upon local factors in the area of embolization (such as age of embolus), and also patients can develop a clinically useful thrombolytic system whether they show marked biochemical changes in response to urokinase therapy or not.

In addition, analysis of the data before infusion indicated decreased levels of plasminogen, fibrinogen, and platelets and increased prothrombin times in 38 (of 140) patients suggesting possible defibrination. Seven $(19 \%)$ of the 38 patients in this group died as compared with three $(3 \%)$ of 102 with more normal laboratory findings before infusion. The incidence of severe haemorrhage was also higher in this group $(38 \%)$ than in the others $(16.5 \%)$

\section{Drug Therapeutic Evaluation in Acute Myocardial Infarction}

ANTHONY P. FLETCHER (Washington University School of Medicine, St Louis, Missouri)

For the last two decades, the statisticaliy controlled therapeutic trial in acute myocardial infarction has involved, largely as a result of British advocacy, only the very simplest of design concepts. Essentially, it has been held that provided all necessary precautions were taken strictly to randomize patients into a study and other precautions taken to avoid bias in patient selection, that the determination of relative mortality between treated and control patient groups would suffice to determine a single drug's therapeutic efficacy and therapeutic value.

Such trial design principles appeal mainly to administrators and statisticians. To the former, since they are simple to understand and relatively easy, though tedious, to organize on a practical basis. To the statisticians, because they feel that in dealing with mortality, they are dealing with 'hard' data and thus, difficulties in statistical analysis of results will be minimized.

However, the experience of the last 25 years, especially with trials of anticoagulant therapy, have shown trials of the conventional type to display many weaknesses. (1) They are extremely inefficient insofar as they utilize only a very small fraction of the data collected. Since only the actual death of a 